\title{
Superación de barreras en la implementación de los protocolos ERAS: aspectos nutricionales
}

\author{
Overcoming barriers in the implementation of ERAS protocols: \\ Nutritional aspects \\ Superação de barreiras na implementação de protocolos ERAS: aspetos nutricionais
}

Paola Sánchez Corrales ${ }^{1 *}$, Esteban Vargas Blanco ${ }^{1}$

Recibido: 21 de julio de 2019. Aceptado para publicación: 21 de agosto de 2019.

Publicado en línea: 9 de noviembre de 2019

https://doi.org/10.35454/rncm.v3n1.022

\begin{abstract}
Resumen
Los protocolos de manejo perioperatorio de ERAS (Enhanced Recovery After Surgery) se basan en la utilización de medidas respaldadas por la evidencia científica actual. Son un conjunto de estrategias multimodales que tienen como objetivo disminuir los tiempos de hospitalización, de recuperación, complicaciones y costos asociados a distintos procedimientos quirúrgicos.

Para su implementación, se requieren intervenciones anestésicas, quirúrgicas, funcionales, nutricionales y de enfermería. Pese a que existe una amplia evidencia que avala su uso en nuestra práctica clínica diaria, existen muchas barreras que han limitado su desarrollo en los diferentes centros hospitalarios. Presentamos una revisión de las limitantes para la aplicación de los componentes nutricionales implicados en estos protocolos, tanto desde la experiencia en la implementación como en fuentes bibliográficas encontradas.

El objetivo de esta revisión es aportar recomendaciones que puedan ser utilizadas en aplicaciones futuras de dichos protocolos en la práctica hospitalaria.
\end{abstract}

Palabras clave: cuidado perioperatorio, nutrición, Enhanced recovery after surgery.

\section{Summary}

The ERAS protocols (Enhanced Recovery After Surgery) have been based on concepts in the utilization of tools in the perioperative period and often supported by scientific evidence.

These are a set of multimodal perioperative strategies that aim to reduce the time of hospitalization, recovery, complications and costs associated with different surgical procedures.

Multiple interventions are required for its implementation: anesthetic, surgical, functional, nutritional and nursing interventions. There is a strong body of evidence supporting its application in the daily practice, but there are multiple barriers that have limited its development in the hospitals. This review intends to highlight the difficulties of the applications of the nutritional components involved in these protocols, not only by experience but also by the scientific evidence.

The objective of this review is to give some recommendations that can be used in these protocols in the daily hospital practice.

Key words: Perioperative care; Nutrition; Enhanced Recovery After Surgery.

\section{Resumo}

Os protocolos de gestão perioperatório ERAS (Enhanced Recovery After Surgery) baseiam-se no uso de medidas apoiadas por evidências científicas atuais. São um conjunto de estratégias multimodais que visam reduzir hospitalizações, tempos de recuperação, complicações e custos associados a diferentes procedimentos cirúrgicos.

Para sua implementação, são necessárias intervenções anestésicas, cirúrgicas, funcionais, nutricionais e de enfermagem. Embora exista ampla evidência que apoie seu uso em nossa prática clínica diária, existem muitas barreiras que limitaram seu desenvolvimento nos diferentes centros hospitalares. Apresentamos uma revisão das limitações para a aplicação dos componentes nutricionais envolvidos nesses protocolos, tanto a partir da experiência na implementação quanto nas fontes bibliográficas encontradas.

O objetivo desta revisão é fornecer recomendações, que possam ser usadas nas futuras aplicações desses protocolos na prática hospitalar.

Palavras-chave: cuidados perioperatórios, nutrição, Enhanced Recovery After Surgery. 


\section{INTRODUCCIÓN}

En la actualidad la implementación de protocolos ERAS $^{\oplus}$ (Enhaced Recovery After Surgery), dirigidos a acelerar el proceso de recuperación posquirúrgica, ha demostrado ser costo eficiente y de gran beneficio para los pacientes que van a ser sometidos a procedimientos quirúrgicos. Por lo anterior, cada vez más centros optan por implementarlos, sin embargo, se enfrentan a una serie de dificultades que entorpecen el proceso y dificultan el cumplimiento. En centros muy bien organizados se reporta un cumplimiento cercano a $75 \%$ y, en general, las razones de incumplimiento la presentan tanto los pacientes como el mismo personal de salud, quienes muchas veces ignoran los protocolos, o simplemente no están de acuerdo con la metodología. Una evaluación realizada en el Reino Unido, reportó que los cirujanos generales perciben que no hay suficiente soporte multidisciplinario en los centros hospitalarios para implementar de forma adecuada el programa, o que la metodología está mal planteada ${ }^{(1,2)}$.

Entre otros puntos a recalcar, que generan algún tipo de desconfianza entre los profesionales es que en algún momento se planteó que el programa ERAS podría aumentar las tasas de reingreso hospitalario, debido a que los pacientes tienen un egreso temprano, aumentando con esto las complicaciones posquirúrgicas, pero esto ha sido descartado con evidencia científica ${ }^{(2)}$.

Los aspectos relacionados con la intervención metabólica y nutricional son uno de los pilares fundamentales en el protocolo ERAS y también deben enfrentar una serie de problemas para lograr su cumplimiento, a pesar de que se ha demostrado que optimizar el estado nutricional en el escenario quirúrugico, mejora el balance nitrogenado y también las alteraciones en el metabolismo de la glucosa ${ }^{(2)}$.

Un estudio publicado por Harnáez et al. en 2016, realizado en pacientes sometidos a cirugía bariátrica indican que las medidas relacionadas con el abordaje nutricional son las que registran el peor cumplimiento, por ejemplo de los 172 pacientes incluidos en el estudio a $90 \%$ se le colocó SNG (sonda nasogástrica) para drenaje intrabdominal, ningún paciente recibió carga de carbohidratos preoperatoria, $100 \%$ recibió profilaxis para náuseas y vómitos postoperatorios; sin embargo, a ningún paciente se inició la alimentación postoperatoria de manera temprana ${ }^{(3)}$.

Tomando en cuenta lo anterior, los aspectos que se detectan como puntos críticos en la implementación del proceso en el campo de la intervención metabólica nutricional son los siguientes:

\section{Coordinación multidisciplinaria}

En algunas ocasiones lograr la coordinación de grupos de expertos en distintas ramas puede parecer una labor titánica; sin embargo, los equipos multidisciplinarios le brindan mayor seguridad al paciente, minimiza los errores médicos, mejora la satisfacción y el rendimiento del personal de salud y disminuye gastos generados por complicaciones y mala praxis $^{(4)}$.

La adecuada coordinación y compañerismo en un equipo hospitalario implementando un protocolo ERAS, ha demostrado mayor efectividad y mejores resultados, que el desarrollo de algunos o pocos parámetros del programa. Esto ha sido ya demostrado estadísticamente, afirmando que los equipos multidisciplinarios tienen mayores beneficios para el paciente quirúrgico $^{(2)}$.

Existe una importante dificultad para el trabajo en equipo entre los diferentes grupos que participan en la implementación de los protocolos ERAS, en su mayoría por el desconocimiento de cada uno de los grupos con respecto a la labor de los otros, lo que hace que se visualicen las mismas, más sencillas o más complejas de lo que en realidad pueden resultar.

Por otro lado, el exceso de trabajo lleva a que exista dificultad para la coordinación de los diversos grupos que permita establecer juntos un trabajo coordinado.

Un aspecto importante que puede obstaculizar la apertura del trabajo en equipo, sobre todo por parte de los cirujanos, es el temor a documentar los resultados obtenidos en las intervenciones quirúrgicas frente a profesionales de otras ramas.

Es importante aclarar que otros profesionales participantes pueden presentar resistencia a la exigencia que representa la participación en este tipo de protocolos en donde se debe actuar en momentos específicos.

Por lo tanto, es indispensable que cada uno de los miembros del equipo conozca a cabalidad su función respectiva, y los momentos de implementación, siendo respetuoso con el campo profesional de cada integrante $y$ entender que cada pieza del equipo es igualmente valiosa. Esto incluye médicos, enfermeros, equipos de soporte nutricional, servicios de nutrición, personal administrativo hospitalario, trabajo social, psicología, fisioterapia, farmacia y personal de sala de operaciones y salones quirúrgicos ${ }^{(2)}$. 


\section{Evaluación nutricional preoperatoria}

La identificación temprana de los pacientes con malnutrición por medio de un método de tamizaje adecuado puede ser un problema cuando no se realiza en forma apropiada. En los hospitales universitarios, por ejemplo, la alta rotación de personal hace que se requieran capacitaciones sistemáticas y entrenamientos continuos. A pesar de que se establece que el tamizaje debe realizarse al ingreso y se reconocen sus beneficios tanto para el paciente como en los costos para el sistema, en Costa Rica no se han oficializado escalas que permitan contar con un formulario estándar de uso obligatorio, dentro de la seguridad social, que es el ente que abarca el grueso de la población. Otro de los inconvenientes es que no existe un tamizaje que haya sido validado para todos los pacientes quirúrgicos y que se considere el estándar de oro, lo que da una amplia gama de elección.

Sin embargo, es claro que la malnutrición preoperatoria ha sido asociada a un incremento de la mortalidad y morbilidad postoperatoria y a un mal pronóstico en los pacientes oncológicos que serán sometidos a cirugía, sobre todo en el tracto gastrointestinal, por lo que estos sistemas de tamizaje permiten priorizar la atención a pacientes que lo requieren, brindándoles la oportunidad de recibir un tratamiento nutricional óptimo previo a la cirugía, que permita corregir las deficiencias encontradas $^{(5)}$.

De las herramientas más utilizadas y más recomendadas en el paciente hospitalizado es el NRS 2002 (Nutritional Risk Screening Score), al cual se le atribuye la capacidad de predecir el riesgo de complicaciones mayores $^{(5)}$. Por otro lado, es importante considerar algunos marcadores bioquímicos como la albúmina, ya que su valor en el período preoperatorio se considera un factor de riesgo de mortalidad y morbilidad y debe considerarse dentro de la evaluación nutricional ${ }^{(5)}$.

Es fundamental sensibilizar a todo el personal de salud y administrativo de los hospitales, con respecto a la importancia de este tema y establecer un sistema estandarizado de inducción y educación continua para todos los profesionales.

\section{Educación al paciente}

En muchas ocasiones se subestima la capacidad del paciente y no se involucra como parte activa de este protocolo. Es importante que el paciente conozca no solo el procedimiento quirúrgico al que va a ser sometido en forma clara y precisa, si no el proceso periope- ratorio en el que se encontrará inmerso y la razón de la participación de diversos equipos, teniendo claros los objetivos del abordaje y comprendiendo todos los posibles escenarios a lo que se puede enfrentar, ya que esto garantiza que el paciente esté mejor preparado ${ }^{(2,6)}$.

Para lograr los objetivos de educación se requiere un cambio cultural del personal de salud, que incluya dentro de su quehacer diario la información al paciente de cada proceso y no solo de los procedimientos. En muchos centros la principal queja del personal es la sobrecarga de trabajo, por lo que algunas de las propuestas es dedicar un profesional en enfermería a la capacitación en el proceso ERAS de cada uno de los pacientes seleccionados ${ }^{(2,7)}$.

A pesar de que algunos administrativos pueden considerar un alto costo asignar personal a este proceso, se ha descrito que los pacientes que dominan el concepto teórico de su cirugía, así como el contexto de procedimientos a los cuales van a ser sometidos, tienen un efecto de "coherencia y control" de su condición actual. Esto promueve un óptimo estado psicológico y emocional del paciente sometido al protocolo, parte vital de un adecuado manejo clínico ${ }^{(2)}$.

Estudios desde hace más de dos décadas, mencionan cómo la consejería efectiva en el preoperatorio se ha asociado a mejoras multidimensionales en estos pacientes, disminución de la ansiedad, recuperación intestinal más rápida, menor necesidad de analgesia, menor estancia hospitalaria e inclusive menos fatiga posquirúrgica. Se resume en que, cuando el paciente tiene expectativas más objetivas de su proceso, esto se manifiesta en mejores resultados en su salud ${ }^{(2,5)}$.

La información que se aporte al paciente debe de ser constante y coherente, además de incluir de manera detallada las vías de alimentación elegidas, tipo de alimentación o suplementación, describir aspectos relacionados con el sabor y textura en caso de utilizar módulos o suplementos, haciendo énfasis en un enfoque terapéutico de la intervención nutricional.

Los medios para brindar esta educación son diversos desde una entrevista personal hasta opciones con multimedia o realidad virtual que permitan una mayor comprensión de cada una de las intervenciones a realizar ${ }^{(7)}$.

\section{Dieta de preparación colónica}

Es usual que los cirujanos que van a intervenir quirúrgicamente a un paciente en colon o recto, opten por la preparación colónica, que incluye entre 5 y 7 días de líquidos claros; sin embargo, hoy se cuenta con eviden- 
cia que establece que hay mayores complicaciones e incrementa el riesgo de malnutrición, práctica que además se asocia a la indicación de preparación mecánica de colon, la cual tampoco posee evidencia suficiente para su respaldo. Las complicaciones de estas prácticas inadecuadas pueden incluir deshidratación preoperatoria, alteraciones hidroelectrolíticas y disconfort del paciente, sin generar ningún tipo de beneficio ${ }^{(5,7)}$.

Romper con esta costumbre tan arraigada es muy dificil, ya que el personal de salud tiende a sentir preocupación por la contaminación fecal durante el tiempo quirúrgico.

El establecimiento de protocolos ERAS elimina este paso innecesario y es fundamental la difusión de este punto entre el personal de salud, para lograr mejores resultados.

\section{Optimización del estado nutricional preoperatorio}

La optimización del estado nutricional es uno de los puntos de mayor complejidad porque involucra aspectos adminitrativos relacionados con rotación de camas y tiempo de hospitalización, entre otros. Algunos de los puntos críticos en este aspecto son:

\section{Evaluación nutricional al ingreso}

La optimización del estado nutricional preoperatoria es un aspecto de vital importancia; sin embargo, uno de los principales inconvenientes tiene que ver con las referencias tardías, cuando el paciente ya presenta una afectación muy grave de su estado nutricional por lo que la instauración de un método de tamizaje como se planteó anteriormente resulta trascendental.

\section{Gastronomía hospitalaria}

Por otro lado, la intervención nutricional en pacientes hospitalizados con alteración del estado nutricional y pobre ingesta, se vuelve complicada debido a una gastronomía hospitalaria precaria e indicaciones inadecuadas en el tipo de dieta, llevando a un mayor deterioro inatrogénico de la condición nutricional del paciente.

La adecuada gestión de los comedores hospitalarios para garantizar no solo calidad nutricional si no también gastronómica resulta vital para implementar cualquier programa que requiera intervención nutrional.

\section{Ayunos preoperatorios}

Es frecuente la indicación de nada por vía oral en los pacientes hospitalizados y se asume como una "normalidad" hospitalaria, por lo que muchas veces se indican o se prolongan de manera innecesaria para la realización de exámenes o procedimientos.

El ayuno preoperatorio prolongado es un punto altamente arraigado no solo en el personal de salud, sino en la población general, que carece de evidencia científica, y se realiza más por una práctica ancestral que rezaba que era indispensable para la prevención de la broncoaspiración durante el procedimiento quirúrgico.

De acuerdo con el protocolo ERAS lo recomendado son 6 horas para sólidos con ingesta de pequeñas cantidades de líquidos libres y ayuno de hasta 2 horas antes de inducción de la anestesia; y carga de carbohidratos la noche anterior y 2 horas antes del procedimiento quirúrgico. Esta intervención ha demostrado una disminución en la resistencia a la insulina de $50 \%$, atenúa las pérdidas postoperatorias de nitrógeno y mejora la función muscular ${ }^{(5,8-11)}$.

\section{Bebidas de carbohidratos}

Las bebidas de carbohidratos son líquidos isotónicos y se recomiendan en el paciente para disminuir la sensación de sed, hambre y ansiedad preoperatoria. Esta intervención también reduce los requerimientos de líquidos en el perioperatorio y la sintomatología postoperatoria en especial náuseas, vómito, mareo, diarrea y dolor, así como la dehiscencia de heridas quirúrgicas ${ }^{(10,11)}$.

Los inconvenientes en la programación de las cirugías y los retrasos que se presentan hacen que sea difícil el cumplimiento de aspectos como la ingesta de maltodextrinas estrictamente 2 horas previas a la cirugía, a pesar de que este ha sido uno de los aspectos que mayores beneficios ha demostrado ${ }^{(10)}$.

Algunos centros han optado por colocar horarios para la administración estándar en la noche y la mañana, lo que les garantiza que la noche anterior y horas cerca a la cirugía aunque no exactamente 2 , el paciente recibirá una carga de carbohidratos, como una medida alterna.

Es importante rescatar que se han establecido criterios de exclusión para el uso de carbohidratos preoperatorios como alteración de la motilidad gastrointestinal, reflujo gastroesofágico severo, obstrucción o carcinoma en la unión esófago-gástrica, pacientes con dificultad 
en el manejo de la vía aérea y pacientes con intoleracia a la maltosa o fructuosa. Se debe considerar que los pacientes con diabetes y neuropatía asociada pueden presentar un retraso en el vaciamiento gástrico y mayor riesgo de aspiración, por lo que guías recientes no recomiendan su uso en pacientes diabéticos. Tampoco se recomienda el uso en cirugías de urgencia ${ }^{(5,12)}$.

\section{Inmunonutrición prequirúrgica}

Un aspecto importante es el uso de inmunonutrición prequirúrgica, la cual está recomendada en guías recientes para su uso oral o enteral y se indica que debe administrarse de 5 a 7 días en todos los pacientes con o sin riesgo de malnutrición que vayan a ser sometidos a cirugía por cáncer del tracto gastrointestinal ${ }^{(13)}$.

Las guías sugieren que la suplementación debe realizarse con L-arginina, L-glutamina, nucleotidos y omega 3, ya que esto ha demostrado disminución de las complicaciones y la estancia hospitalaria ${ }^{(5)}$.

Este punto presenta varias dificultades para los servicios, incluyendo el costo de la inmunonutrición, el seguimiento y verificación del consumo del mismo, sobre todo cuando se administra vía oral y la tolerancia y aceptación por parte del paciente debido especialmente a su sabor poco agradable.

En este punto la educación al personal de salud vuelve a resultar vital para que se colabore en la vigilancia y administración del mismo.

En lo que respecta al paciente es fundamental involucrarlo en el proceso y explicarle la importancia del suplemento y el impacto que este medicamento puede tener en su recuperación futura.

\section{Eliminar el consumo de alcohol}

Diversos estudios sugieren que el abuso de alcohol incrementa la morbilidad postoperatoria sobre todo relacionada con infecciones. Se recomienda suspender la ingesta de alcohol 4 semanas previas a la cirugía ${ }^{(5)}$. El monitoreo de recaídas es complejo y compromete al propio paciente, siendo la educación y concentientización del paciente la única herramienta posible de utilizar.

\section{Uso de suplementos nutricionales}

Está descrito que la pérdida de peso no intencional mayor a $5 \%$ - $10 \%$ incrementa el riesgo de complicaciones, por lo que en los pacientes con alto riesgo de malnutrición se debe considerar la suplementación oral y adicionar parenteral cuando sea necesario y esta debe brindarse por al menos 7 - 10 días para disminuir el riesgo de infecciones y complicaciones de fugas de anastomosis, incluso se recomienda continuar estos en el postoperatorio hasta tanto el paciente alcance al menos $60 \%$ de su requerimiento ${ }^{(5,13)}$.

En este punto es importante detenerse a comentar sobre la dificultad que se presenta frente a la tolerancia y aceptación de los suplementos por parte de los pacientes, por lo que el personal de salud debe explicar al paciente que es una parte trascendental del proceso terapéutico.

Es además frecuente que personal administrativo presione para mejorar la rotación de camas y considere que no es posible retrasar la cirugía por los costos implicados, más en este aspecto es fundamental hacer ver el costo beneficio de la intervención y proponer metodología de manejo ambulatoria.

\section{Reanudación de la ingesta postoperatoria}

Este es uno de aspectos más críticos, debido a la arraigada creencia de que la presencia de nutrientes en el tracto gastrointestinal puede ser deletérea y afectar los resultados de la intervención quirúrgica, lo anterior a pesar de que se ha demostrado en la literatura que al contrario, el inicio temprano de la alimentación produce una mejor evolución y una recuperación más acelerada ${ }^{(7,12)}$.

Investigaciones han demostrado que la alimentación se puede tolerar en el primer día postoperatorio, que la ingesta revierte la atrofia mucosa inducida por el ayuno y que se incrementa el contenido de colágeno en la anastomosis y su resistencia. Además la ingesta temprana propicia un balance nitrogenado positivo, disminuye la resistencia a la insulina, favorece la cicatrización de heridas y reduce el riesgo de sepsis, y de formación de fístulas ${ }^{(7,13)}$.

La mejora en la sintomatología también se ha presentado con la aplicación de los protocolos ERAS que incluyen el inicio temprano de la dieta, la fatiga postoperatoria, asociada a pérdida de masa y función muscular y peor respuesta cardiovascular al ejercicio, mejora cuando se aplica este protocolo ${ }^{(7)}$.

También se documenta una disminución en las náuseas postoperatorias, una recuperación rápida de la función intestinal y una disminución de la estancia hospitalaria, incluso se establece que la ingesta espontánea rara vez supera las 1.500 calorías, por lo que debe considerarse la suplementación ${ }^{(5)}$.

Las recomendaciones incluyen el inicio de dieta líquida a demanda en 4 horas postoperatorias y 2 bebi- 
das proteicas, se sugiere que si la tolerancia es buena se ofrezca dieta semiblanda en la cena. En el día 1 postoperatorio se recomienda dieta a libre elección y 3 bebidas proteicas, para el día 4 dieta normal ${ }^{(7)}$.

\section{Evitar sobrecarga hídrica}

El aporte adecuado de líquidos durante el perioperatorio resulta en una de las medidas con mayor dificultad para su cumplimiento, sobre todo porque existe la creencia errónea de que el paciente hospitalizado siempre debe tener líquidos intravenosos indicados durante la hospitalización ${ }^{(1,10)}$.

Estudios demuestran que por cada litro de líquido dado durante el día de la cirugía se incrementa el riesgo de síntomas postoperatorios que retrasan la recuperación hasta en $32 \%$, en especial por su impacto en el sistema cardiorrespiratorio. También puede provocar edema periférico y visceral, así como anormalidades electrolíticas mientras que la hipovolemia puede afectar el gasto cardíaco y la oxigenación tisular ${ }^{(8,10,12)}$.

El objetivo es mantener la euvolemia, ya que esta produce menos complicaciones postoperatorias, un retorno más temprano de la función intestinal y una estancia hospitalaria más corta, para lograrlo se requiere un balance de líquidos neutro ${ }^{(5,8,10)}$. Lograr la euvolemia exige un estricto manejo de líquidos en el preoperatorio, intraoperatorio y postoperatorio. Como se explicó anteriormente, las pacientes deben ingerir líquidos claros o una bebida con carbohidratos 2 horas antes de la inducción anestésica, estos líquidos deben ser cuantificados dentro de los aportes, algo que con frecuencia se omite ${ }^{(14)}$.

En el postoperatorio, se recomienda la transición rápida a la ingesta oral y suspensión de líquidos por vía intravenosa. Si los pacientes necesitan líquidos intravenosos, estos deben ser bajos en sodio y volumen acorde con requerimientos con el fin de mantener el balance en cero en el menor tiempo posible ${ }^{(14)}$.

Por este motivo, se debe realizar una hidratación ajustada a las pérdidas hemáticas e insensibles. Se recomienda la administración de un volumen fijo de cristaloides para el mantenimiento del balance hídrico; y quedan reservados los coloides en caso de inestabilidad hemodinámica, así como para reemplazar las pérdidas hemáticas ${ }^{(5,9)}$.

\section{Evitar el uso de sonda nasogástrica}

La sonda nasogástrica (SNG) ha sido utilizada de forma rutinaria en la cirugía abdominal con el objetivo de logar descompresión gástrica para la prevención de la distensión abdominal, las náuseas y el vómito; sin embargo, esta práctica no está sustentada en la literatura, por el contrario, parece que su uso retrasa la aparición del tránsito intestinal, prolonga la estancia hospitalaria e incrementa las complicaciones respiratorias postoperatorias, además de producir gran incomodidad en el paciente ${ }^{(5,9,12)}$.

En la actualidad es frecuente observar estas prácticas que entorpecen la ingesta precoz como algo instaurado en los centros de salud, por lo que es fundamental que estas no se pongan de manera rutinaria, y si hay una indicación precisa, se suspendan en sala de operaciones cuando termine el procedimiento quirúrgico.

\section{Prevención de náuseas y vómito}

El control de náuseas y vómito resulta trascendental para lograr una adecuada ingesta en el paciente, por lo que es importante establecer los factores de riesgo mediante la Escala Apfel (Tabla 1), aplicar profilaxis farmacológica intraoperatoria, adoptar medidas de reducción del riesgo y disminuir el uso de derivados de opiáceos durante el postoperatorio ${ }^{(12)}$.

Tabla 1. Escala Apfel

\begin{tabular}{|l|c|}
\hline \multicolumn{1}{|c|}{ Factor de riesgo } & Puntos \\
\hline Mujer & 1 \\
\hline No fumadora & 1 \\
\hline Historia de náuseas /vómito en el postoperatorio & 1 \\
\hline Opioides en el postoperatorio & 1 \\
\hline Puntos & $0-4$ \\
\hline
\end{tabular}

\begin{tabular}{|c|c|c|c|c|}
\hline \multirow{2}{*}{$\begin{array}{l}\text { Estimación del } \\
\text { riesgo de PONV }\end{array}$} & 1 & 2 & 3 & 4 \\
\hline & $20 \%$ & $40 \%$ & $60 \%$ & $80 \%$ \\
\hline
\end{tabular}

PONV: náuseas y vómito postperatorios Adaptado de: Ramírez R. 2013 ${ }^{(16)}$

Las estrategias requeridas para disminuir el riesgo no se aplican, ya que muchos profesionales desconocen esta escala de evaluación, por lo que la divulgación y concientización resultan indispensables para garantizar una ingesta postoperatoria temprana.

\section{Prevención del íleo}

El uso de algunos fármacos puede favorecer una recuperación más rápida de la función inestinal; por 
Tabla 2. Recomendaciones para la implementación de aspectos nutricionales de los protocolos ERAS

\begin{tabular}{|c|c|}
\hline Punto crítico & Recomendaciones \\
\hline $\begin{array}{l}\text { Educación del personal } \\
\text { de salud }\end{array}$ & $\begin{array}{l}\text { - Establecer un equipo interdisciplinario para el abordaje de los protocolos ERAS y posteriormente } \\
\text { se debe crear un sistema de educación y difusión continua que permita conocer aspectos técnicos, } \\
\text { logísticos y fisiopatológicos de cada uno de los puntos involucrados en el protocolo, para que de esta } \\
\text { manera todos comprendan correctamente los procesos y se articule el protocolo en forma adecuada. } \\
\text { - Educar al personal de salud sobre los procedimientos correctos en la preparación de pacientes que } \\
\text { van a ser sometidos a cirugía colorrectal. } \\
\text { - Desmitificar mediante procesos de educación aspectos relacionados con inicio temprano de la } \\
\text { alimentación y el uso de sondas nasogástricas. }\end{array}$ \\
\hline $\begin{array}{l}\text { Intervención } \\
\text { preoperatoria temprana }\end{array}$ & $\begin{array}{l}\text { - Estandarizar un método de tamizaje nutricional al ingreso en el paciente quirúrgico y establecer un } \\
\text { mecanismo para obligar su aplicación. } \\
\text { - Intervenir, mediante educación y políticas institucionales, puntos críticos que mejoren el estado } \\
\text { nutricional prequirúrgico: mejora en aspectos relacionados con la gastronomía hospitalaria, evitar } \\
\text { ayunos innecesarios, uso de suplementación e inmununutrición y bebidas de carbohidratos } \\
\text { prequirúrgicas. }\end{array}$ \\
\hline Educación al paciente & $\begin{array}{l}\text { - Concientizar sobre la necesidad de educar al paciente en el proceso y destinar tiempo de enfermería } \\
\text { para esta labor. }\end{array}$ \\
\hline $\begin{array}{l}\text { Monitoreo del aporte de } \\
\text { fluidos perioperatorios }\end{array}$ & $\begin{array}{l}\text { - Establecer protocolos para la administración de fluidos en los periodos pre, intra y postoperatorio que } \\
\text { garantice un aporte adecuado de los mismos y minimice riesgos. }\end{array}$ \\
\hline $\begin{array}{l}\text { Prevención de náuseas y } \\
\text { vómito perioperatorios }\end{array}$ & $\begin{array}{l}\text { - PONV. } \\
\text { - Gestionar la inclusión de mayor variedad de analgésicos no opioides y educar para el uso adecuado } \\
\text { de los mismos. }\end{array}$ \\
\hline Auditorias & $\begin{array}{l}\text { - Ajustar la aplicación de los protocolos ERAS a las realidades institucionales que permita mediante } \\
\text { el establecimiento de diversos sistemas y con ayudas tecnológicas las auditorias constantes para } \\
\text { mejorar los diversos aspectos que presenten puntos críticos. }\end{array}$ \\
\hline
\end{tabular}

ejemplo, la metoclopramida tiene efecto agonista colinérgico y antagonista de los receptores de dopamina, con un mayor efecto en el tracto digestivo superior y poco efecto en la motilidad colorrectal ${ }^{(7)}$.

Las guías refieren que un abordaje multimodal minimiza el riesgo de desarrollar íleo en el postoperatorio, y este incluye: limitar el uso de opiodes, y técnicas quirúrgicas mínimamente invasivas, eliminar SNG y control hídrico. También se recomienda mascar chicle, bisacodyl, óxido de magnesio y café( ${ }^{(5)}$.

La principal dificultad en este punto tiene que ver con la recomendación de abolir el uso de opioides en el postoperatorio, debido a la limitación en las posibilidades de analgesia en los sistemas de salud y por conductas aprendidas muy arraigadas.

\section{Auditorías}

Los protocolos ERAS están compuestos por una serie de elementos, por lo que las auditorías resultan complejas $^{(15)}$.
Una evaluación constante de los puntos de aplicación de los protocolos ERAS es fundamental para garantizar el éxito, esto debe incluir resultados en pacientes y realizar una retroalimentación que sea la base de las mejoras $^{(1)}$. El apoyo en base de datos y elementos tecnológicos que permitan un adecuado seguimiento de los pacientes con el objetivo de auditar posteriormente puede resultar muy provechoso.

\section{CONCLUSIÓN}

En conclusión la implementación de los protocolos ERAS en general y de los aspectos relacionados con nutrición en particular, representa un reto para todos los sistemas de salud. Sin embargo, los beneficios reportados justifican y casi exigen su uso. Es por esto que resulta fundamental identificar puntos críticos que permitan evaluar diversas opciones para lograr la aplicación de los mismos, de acuerdo con las características propias de los centros y la experiencia de hospitales con condiciones similares que permitan agilizar los procesos. 


\section{Financiación}

El presente artículo no tuvo financiación.

\section{Conflicto de intereses}

Los autores declaran no tener conflicto de intereses.

\section{Declaración de autoría}

Los autores participaron por igual en la redacción del artículo; también revisaron y validaron su versión final.

\section{Referencias bibliográficas}

1. Abeles A, Kwasnicki R, Darzi A. Enhanced recovery after surgery: Current research insights and future direction. World J Gastrointest Surg. 2017;27 9(2):37-45.

2. Kahokehr A, Sammour T, Zargar-Shoshtari K, Thompson L, Hill A. Implementation of ERAS and how to overcome the barriers. International Journal of Surgery. 2009;7:16-9.

3. Hernáez A, Redondo E, Allue M, Navarro A, Calvo B, Bielsa M. Dificultades en la implementación de un protocolo de recuperación intensificada en cirugía baríatrica. Bariátrica y Metabólica Iberoamérica. 2016;(6)3:1245-8.

4. Peedziwiatr M, Mavrikis J, Witowski J, Adamos A, Major P, Nowakowski M, Budzynski A. Current status of enhanced recovery after surgery (ERAS) protocol in gastrointestinal surgery. Medical Oncology. 2018;35:95.

5. Gustafsson U, Scott M, Hubner M, Nygren NN, Francis N, Rockall T, Young-Fadok T, Hill A, Soop M, de Boer H, Urman R, Chang J, Fichera J, Kessler H, Grass F, Whang E, Fawcett W, Carli F, Lobo D, Rollins K, Balfour A, Baldini G, Riedel B, Ljungqvist O. Guidelines for Perioperative Care in Elective Colorectal Surgery: Enhanced Recovery After Surgery (ERAS) Society Recommendations: 2018. World J Surg. 2019;43:659-95.

6. Rinninella E, Persiani R, D’Ugo D, Pennestri F, Cicchetti A, Di Brino E, Cintoni M, Abele G, Miggiano D, Gasbarrini A,
Mele M. NutriCatt protocol in the Enhanced Recovery After Surgery (ERAS) program for colorectal surgery: The nutritional support improves clinical and cost-effectiveness outcomes. Nutrition. 2018;50:74-81.

7. Roig J, Rodríguez R, García J, Villalba F, Salvador A, Sancho C, Albors P, Puchades F, Fuster C. Rehabilitación multimodal en cirugía colorrectal. Sobre la resistencia al cambio en cirugía y las demandas de la sociedad. Cir. Esp. 2007;81(6):307-15.

8. Elizondo S. Nutrición en el perioperatorio, protocolo ERAS. Medicina Crítica. 2016;39(1):158-9.

9. Carrillo R, Espinoza de los Monteros I, Pérez A. Una nueva propuesta de la medicina perioperatoria. El protocolo ERAS. 2013;36(Supl.1):S296-01.

10. Gustafsson UMD, Hausel J, Thorell A, Ljungqvist O, Soop M, Nygren NN. Adherence to the Enhanced Recovery After Surgery Protocol and Outcomes After Colorectal Cancer Surgery. Arch Surg. 2011;146(5):571-7.

11. Sánchez A, Papaprieto K. Nutrición preioperatorioa en protocolos quirúrgicos para una mejor recuperación postoperatorio (Protocolo ERAS). Rev Med Chile. 2017;145:1447-53.

12. Sánchez-Urdazpal L, Salido S, Alday E, Gómez L, Molina B. Implantación de un programa ERAS en cirugía hepática. Nutr Hosp. 2015;31(5):16-29.

13. Mariette C. Role of the nutritional support in the ERAS programme. Journal of Visceral Surgery. 2015;152(1):S18-20.

14. Carey E, Moulder J. Manejo perioperatorio e implementación de programas de mejoramiento de la recuperación en cirugía ginecológica para indicaciones benignas. Obstet Gynecol. 2018;132:137-46.

15. Köhnenkampf R, Maldonado F. Protocolos de recuperación acelerada después de cirugía itienen espacio en nuestra práctica diaria actual? Enhanced Recovery After Surgery (ERAS) protocols zis there a place in our clinical practice? Rev Chil Anest. 2019;48:20-7.

16. Ramírez R. Náuseas y vómito en el perioperatorio. Un abordaje integral y factores de riesgo. Revista Mexicana de Anestesiología. 2013;36(2):346-7. 